\title{
Establishment of Dose Reference Levels for Nuclear Medicine in Sudan
}

\author{
Wadah M. Ali1,2*, Ruba Mohamed Elawad³, Mohmmed Alhadi A. Ibrahim ${ }^{4}$ \\ ${ }^{1}$ Medical Imaging Department, College of Allied Medical Science, Gulf Medical University, Ajman, UAE \\ ${ }^{2}$ Nuclear Medicine Department, Radiological Science and Nuclear Medicine College, The National Ribat University, Khartoum, Sudan \\ ${ }^{3}$ Radiology Department, Madina Medical Center, Madina City, KSA \\ ${ }^{4}$ Nuclear Medicine Department, Radiation and Isotopes Center of Khartoum, Khartoum, Sudan \\ Email: ^dr.wadah@gmu.ac.ae, *waddahandwaddaha@gmail.com
}

How to cite this paper: Ali, W.M., Elawad, R.M. and Ibrahim, M.A.A. (2016) Establishment of Dose Reference Levels for Nuclear Medicine in Sudan. Open Journal of Radiology, 6, 258-263.

http://dx.doi.org/10.4236/ojrad.2016.64034

Received: September 14, 2016

Accepted: October 15, 2016

Published: October 18, 2016

Copyright $\odot 2016$ by authors and Scientific Research Publishing Inc. This work is licensed under the Creative Commons Attribution International License (CC BY 4.0).

http://creativecommons.org/licenses/by/4.0/ (c) (i) Open Access

\begin{abstract}
In this study, a national survey for establishment of Nuclear Medicine (NM) Dose Reference Levels (DRLs) for adult patients was carried out. The Administrated Activity (AAs) (MBq) was collected from six nuclear medicine departments. Factors influencing the image quality were also observed. The established Sudan National DRLs represent the AA value corresponding to 75th percentile of the AA frequency distribution. Generally, Sudan National DRLs and average AAs are comparable with the papers published in the international literature. All Sudanese DRLs values were found within the international range. While it is noted that the Sudanese DRLs is higher than the values of ARSA except for the MIBI pharmaceuticals that used in both parathyroid and myocardial perfusion scan and for ${ }^{99 \mathrm{~m}} \mathrm{TcDTPA}$ that used for Dynamic Renal scan study the DRLs values were decreased. In compared with UNSCEAR 2008 data, the average dose $(\mathrm{MBq})$ for Sudanese we note that the bone scan falls within the average values while it's lower in all other scans except for parathyroid scan in which the AAAs increase more than twice. When compared to BSS 1996, it showed variation in increased and decreased AAAs. There may be potential for reducing the higher values of AAs, in co-operation with Nuclear Medicine staff.
\end{abstract}

\section{Keywords}

Nuclear Medicine, Diagnostic Reference Level DRLs

\section{Introduction}

Diagnostic reference Levels (DRLs) have been introduced by the International Com- 
mission on Radiological Protection ICRP publication 60 [1] and 37 [2] and by European Directive 97/43/Euratom [3] for assisting the optimization of radiological investigation. The objective of DRLs is to help avoid radiation dose to the patient that does not contribute to the clinical purpose of a medical imaging task. This accomplished by comparison between the numerical values of the DRLs (delivered from relevant regional, national of local data) and the mean or other appropriate value observed in practice for a suitable reference group of patient or a suitable reference phantom. All DRLs have been given in term of Administrated Activity (AA) in Mega Becquerel (MBq). There is a large variation between DRLs given by countries. DRLs in NM are based on AAs used for normal size patients (typically $70 \pm 15 \mathrm{Kg}$ ). The concept of DRLs is not based on the 75th percentile but on the AA necessary for good image quality during a standard procedure. Committee 3 of ICRP encourages authorized bodies to set DRLs that best meet their specific needs and that are consistent for the regional, national or local area to which they apply [4]. In nuclear medicine, the effective dose is directly proportional to AA. Therefore, it is highly important to give guidance for a dosage and the following effective dose, especially concerning pediatric patients [5].

\section{Method}

The data of this study were collected by complying the checklist that concerning the administrated activities (AAs) $(\mathrm{MBq})$ to the standard sized adult patients (i.e. $70 \pm 10$ $\mathrm{Kg}$ ) for standard procedure necessary to obtain the optimum diagnostic information as recommended by previous study [6]. The data were collected from six nuclear medicine departments (100\% of total Number of nuclear medicine Departments in Sudan). i.e. this study covers all the nuclear medicine activities in Sudan without Exclusion to any units or department. administrated activities frequency distribution were obtained for the nuclear medicine diagnostic scan presented in Table 1 and Table 2 and in Figure 1 with comparing the values to the ARSAC [7], UNSCEAR 2009 A [8], and BSS 1996 B [9]. For each patient the dose was calculated according to the patients weight using the formula of patient dose for exam $=($ slandered dose of exam $\times$ patient weight $) /$ standard weight $(70 \mathrm{Kg})$, and this is a fastest methods for obtaining the patient dose on routine work rather than the use of body Mass index (BMI). Sudan National DRLs were accomplished according to which the DRLs represent the administrated activities value corresponding to the 75 th percentile of the distribution. Also, the average AAs (AAAs) and the relevant ranges were calculated.

$\mathrm{A}=$ Typical AAs.

$\mathrm{B}=$ Guidance levels (maximum usual activities).

\section{Result}

Established Sudan DRLs (i.e. national) are presented in Table 1 and Figure 2. The Sudanese AAAs (i.e. national) are presented in Table 2 and Figure 3. Factors, such as instrumentation's poor performance and procedures followed by the staff, which influence the image quality and may result in higher AAs, were also investigated during the 
Table 1. Sudan DRLs (values are A in MBq, for adults).

\begin{tabular}{cccc}
\hline Radioisotopes study & $\begin{array}{c}\text { Sudan DRLs } \\
\text { (calculated) }\end{array}$ & $\begin{array}{c}\text { Most common } \\
\text { values ARSA [7] }\end{array}$ & $\begin{array}{c}\text { International } \\
\text { range [5] }\end{array}$ \\
\hline${ }^{99 \mathrm{~m} \text { Tc-MDP Bone scan }}$ & 777 & $600(800$ SPECT) & $500-1110$ \\
${ }^{99 m}$ TcO4 Thyroid scan & 185 & 80 & $75-222$ \\
${ }^{99 m}$ Tc-DMSA Static renal scan & 173.9 & 80 & $70-183$ \\
99m Tc-DTPA Dynamic renal scan & 206.5 & 300 & $150-540$ \\
${ }^{99 m}$ Tc-MIBI Parathyroid scan & 555 & 600 & $400-900$ \\
${ }^{99 m}$ Tc-MIBI myocardial perfusion imaging* & 740 & 1200 & $300-1480^{*}$ \\
\hline
\end{tabular}

${ }^{*}=$ one day protocol, $\mathrm{MDP}=$ methylene-diphosphonate, DMSA $=$ Dimercaptosuccinate, DTPA = Diethylene Triamine Penta Acidic Acid, MIBI = methoxyisobutylisonitrile.

Table 2. Sudan AAA (values are A in MBq, for adults).

\begin{tabular}{cccc}
\hline Radioisotopes study & Sudan AAA & UNSCEAR 2008 A [8] & BSS 1996 B [9] \\
\hline${ }^{99 m}$ Tc-MDP Bone scan & 709.7 & $740-1110$ & 600 \\
${ }^{99 m}$ TcO4 Thyroid scan & 184.3 & 370 & 200 \\
${ }^{99}$ Tc-DMSA Static renal scan & 165.2 & 185 & 160 \\
${ }^{99 m}$ Tc-DTPA Dynamic renal scan & 301.3 & 370 & 350 \\
${ }^{99 m}$ Tc-MIBI Parathyroid scan & 555 & 200 & $75-150$ \\
${ }^{99 m}$ Tc-MIBI myocardial perfusion imaging* & 708.3 & 1100 & Not identified \\
\hline
\end{tabular}

A = Typical AAs, B = Guidance levels (maximum usual activities).

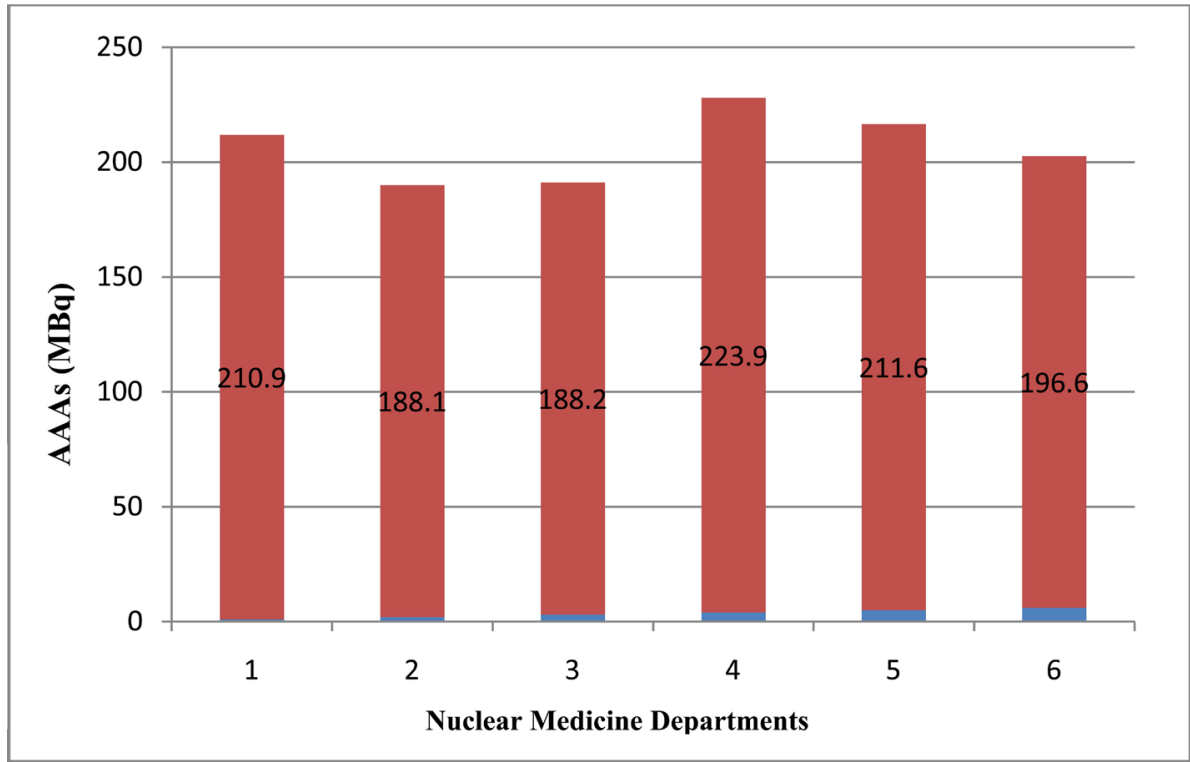

Figure 1. AAAs frequency distribution for Dynamic Renal scan with ${ }^{99 \mathrm{~m}}$ Tc-DTPA AAAs $=$ Average Administrated Activities.

survey and found satisfactory. Figure 1 represents the AAAs of one example of nuclear medicine scan that was commonly performed in Sudan which is ${ }^{99 \mathrm{~m}} \mathrm{Tc}$-DTPAuse for 


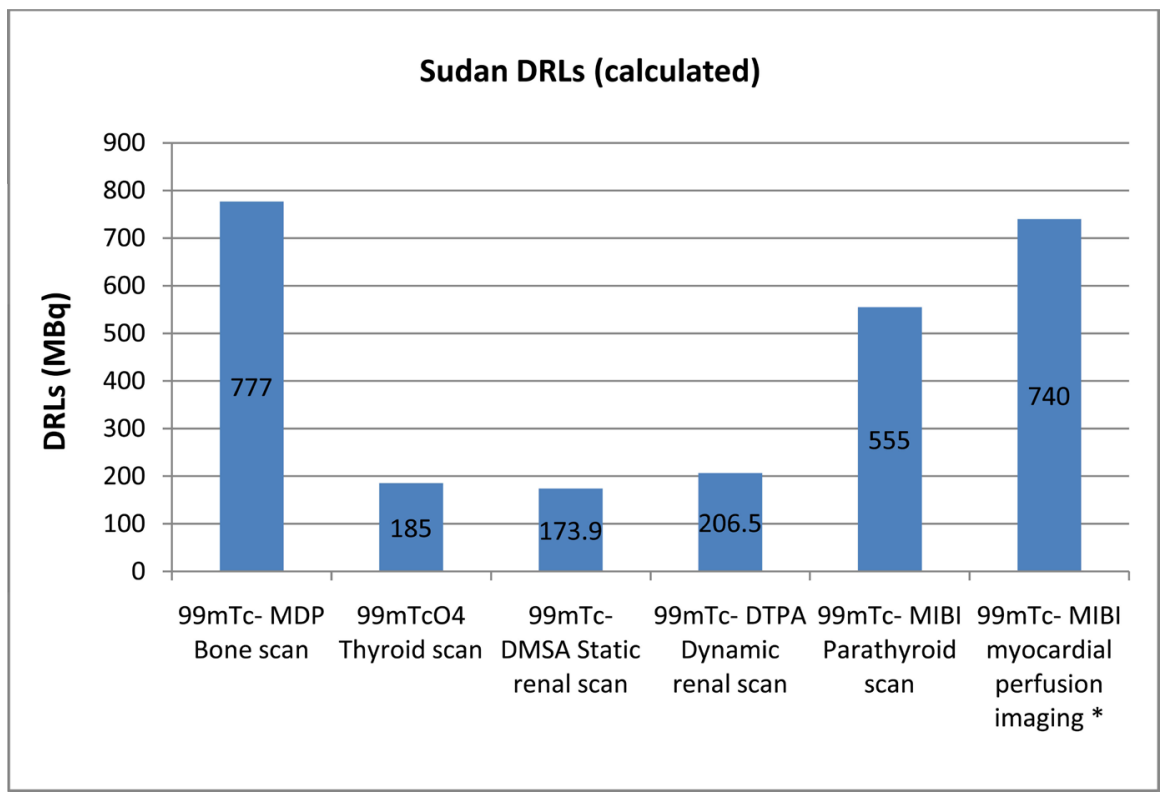

Figure 2. The calculated Sudan DRLs for nuclear medicine examination.

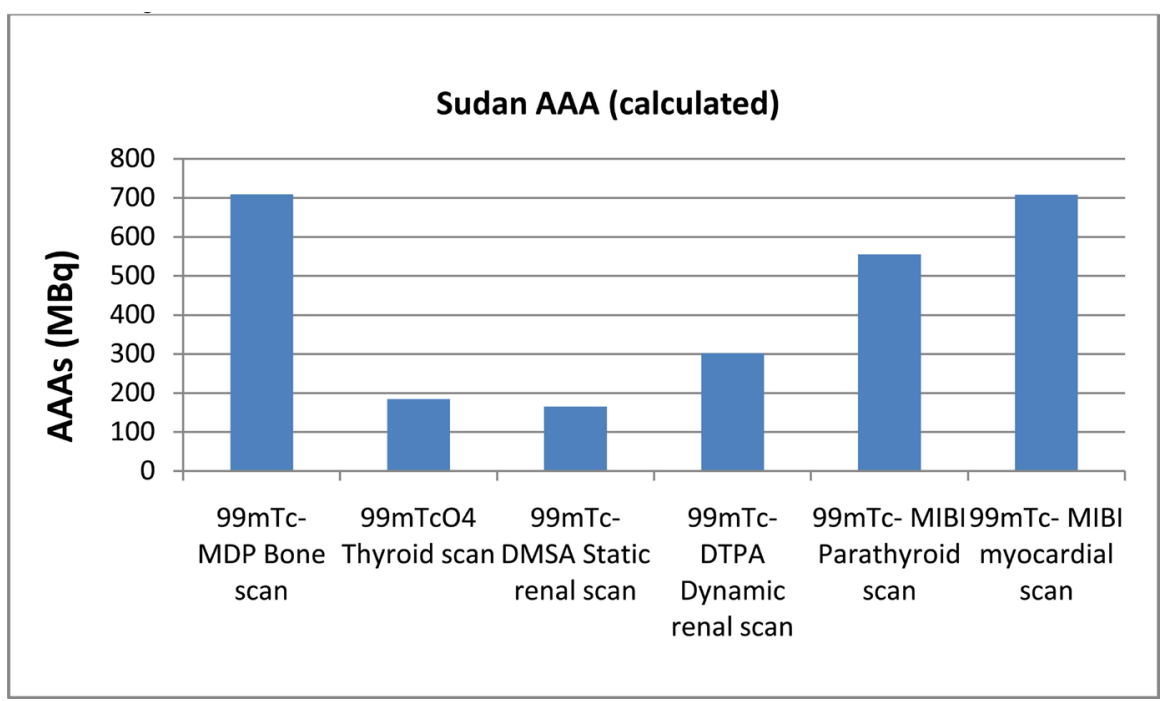

Figure 3. The calculated Sudan AAAs for nuclear medicine examination.

renal system function evaluation. We noted clearly that the range of AAAs of DTPA range from 188.1 to $223.9 \mathrm{MBq}$ with no matching values between the departments because the AAAs depend on the department protocol and the averaged of patients scanned per unit time.

\section{Discussion}

\subsection{Sudan DRLs}

All Sudanese DRLs values were found within the international range [5] as they showed in Table 1, the Sudan DRLs for Bone scan, Thyroid Scan, Static renal scan, Dynamic Renal scan, Parathyroid scan, and Myocardial scan are 777, 185, 173.9, 206.5, 555 and 
$740 \mathrm{MBq}$ respectively compare to international range of (500 - 1110), (75 - 222), (70 183), (150 - 540), (400 - 900), and (300 - 1480) MBq respectively. While it is noted that the Sudanese DRLs is higher than the values of ARSA [7] except for the MIBI pharmaceuticals that used in both parathyroid and myocardial perfusion scan and for ${ }^{99 \mathrm{~m}}$ TcDTPA that used for Dynamic Renal scan Study the DRLs values were decreased. Opposite to ARSA, the nuclear medicine centers and activities in Sudan was newly introduced, and for the reasons of Socio-economic factors somehow the dose in sometimes was not optimized even with a final diagnosable image.

\subsection{Sudan AAAs}

In compared with UNSCEAR 2008 data [8] the average dose (MBq) for Sudanese we note that the bone scan falls within the average values while its lower in all other scans except for parathyroid scan AAAs which increase more than twice (555 MBq compared to $200 \mathrm{MBq}$ ) as the technologist used high dose for optimum detection of the pathology of the small sixed parathyroid gland because of the lack of high resolution camera in some center that must be used for imaging small organs and gland like parathyroid, but as DRLs the parathyroid gland value is still within the acceptable range of international range [5] [400 - $900 \mathrm{MBq}$ ] and also the values was still lower than that of ARSA [8] [600 $\mathrm{MBq}]$. In Routine work in nuclear medicine departments in which the parathyroid scan carried out the technologist use the dose increase by a factors of 1.5 to 2 from a measurable dose using patient weight is provide an optimum image quality, the experiments of this values was carried out earlier with the feedback of nuclear medicine specialist about the influence if increasing the radiation dose on the image quality. While when compared to BSS 1996 [9] it showed variation in increased and decreased AAAs.

For establishments of DRLs the 75th percentile methods will use, while in case of future re-evaluation an "optimum values" is recommended to be use in NM DRLs instead of 75 th percentile [10].

\section{Conclusions}

In some studies, Sudan DRLs and AAAs appear lower than the values found in literature while in other cases observed higher.

Meeting the DRLs does not automatically mean that good practice is performed [8]. There is a minimum activity for each radiopharmaceuticals and a baseline activity that is multiplied with factor given in tables according to the patient weight [5]. Sudan DRLs for nuclear Medicine Diagnostic studies are to be as national guideline and should not be exceeded only for individual patients who are over standard weight.

\section{References}

[1] International Commission on Radiological Protection. Recommendation of the Annals of the ICRP 1991, 1-3.

[2] International Commission on Radiological Protection. Radiological Protection and Safety in Medicine ICRP Publication 73. 
[3] (1997) European Commission. Council Directive 97/43/EURATOM of 30 June 1997 on Health Protection of Individuals against the Danger of Ionizing Radiation in Relation to Medical Exposure. Official Journal of the European Commission, 180.

[4] International Commission on Radiological Protection ICRP. Diagnostic Reference Levels in Medical Imaging. Review and Additional Advice.

[5] Diagnostic Reference Levels for Nuclear Medicine Procedure, DRLs in Thirty-Sex European Countries, DDM2 Survey (2011).

[6] Vogiatzi, S., Kipouros, P. and Chobis, M. (2011) Establishment of Dose Reference Levels for Nuclear Medicine in GREECE. Radiation Protection Dosimetry, 147, 237-239.

[7] A Web Module Produced by Committee 3 of the ICRP.

[8] Administration of Radioactive Substances Advisory Committee. Note for Guidance on the Clinical Administration of Radiopharmaceuticals and Use of /Sealed Radioactive Source. ARSAC (2006).

[9] European Commission. Guidance on Diagnostic Reference Levels (DRLs) for Medical Exposure. EC Radiation 109. European Commission (1999).

[10] Giannopoulous, H. and Housianakou, I. (2000) Evereday Practice Protocols in Nuclear Medicine.

\section{Submit or recommend next manuscript to SCIRP and we will provide best service} for you:

Accepting pre-submission inquiries through Email, Facebook, LinkedIn, Twitter, etc. A wide selection of journals (inclusive of 9 subjects, more than 200 journals)

Providing 24-hour high-quality service

User-friendly online submission system

Fair and swift peer-review system

Efficient typesetting and proofreading procedure

Display of the result of downloads and visits, as well as the number of cited articles

Maximum dissemination of your research work

Submit your manuscript at: http://papersubmission.scirp.org/

Or contact ojrad@scirp.org 